\title{
Immunohistochemical evaluation of tumor budding for stratifying T1 colorectal cancer: optimal cut-off value and a novel computer- assisted semiautomatic method
}

\author{
Manabu Takamatsu $\mathbb{D}^{1} \cdot$ Hiroshi Kawachi $\mathbb{D}^{1} \cdot$ Noriko Yamamoto $^{1} \cdot$ Maki Kobayashi $^{1} \cdot$ Yuka Toyama $^{1}$. \\ Takashi Maekawa' ${ }^{1}$ Akiko Chino $^{2} \cdot$ Shoichi Saito $^{2} \cdot$ Masashi Ueno $^{3} \cdot$ Yutaka Takazawa $^{1} \cdot$ Yuichi Ishikawa $^{1}$
}

Received: 27 August 2018 / Revised: 23 October 2018 / Accepted: 16 November 2018 / Published online: 14 December 2018

(c) United States \& Canadian Academy of Pathology 2018

\begin{abstract}
High-grade tumor budding is an adverse prognostic factor for submucosal invasive (T1) colorectal cancer used to predict the risk for lymph node metastasis in endoscopically resected specimens. Cytokeratin immunohistochemistry is a potential option for evaluating tumor budding. The optimal cut-off value between low- and high-grade budding has not yet been determined, however, and the high inter-observer variability in selecting budding foci remains problematic. We explored the optimal cut-off value for predicting lymph node metastasis using cytokeratin immunohistochemistry, and developed a novel computer-assisted semiautomatic quantification method to reduce inter-observer variability. A retrospective single-institution study of $463 \mathrm{~T} 1$ colorectal cancer cases was conducted. Cases were split into derivation and validation datasets. Tumor budding foci were counted manually and semiautomatically using Image J software on cytokeratin immunohistochemistrystained specimens. We determined the cut-off values and compared inter-observer variability among pathologists between the two methods. Univariate and multivariate analyses of the derivation dataset were performed to select the risk factors for lymph node metastasis. Predictive simulation for the validation dataset was conducted. The optimal cut-off values for the manual and semiautomatic methods were $\geq 10$ and $\geq 12$, respectively. For both methods, multivariate analyses revealed that venous invasion, lymphatic invasion, and high-grade tumor budding were independent risk factors for lymph node metastasis. The semiautomatic method provided significantly better inter-observer agreement. The predictive and observed lymph node metastasis frequencies were highly correlated in the validation dataset.
\end{abstract}

\section{Introduction}

Endoscopic treatment for colorectal cancer confined to the mucosa (Tis) and invading the submucosa (T1) has

Supplementary information The online version of this article (https:// doi.org/10.1038/s41379-018-0189-1) contains supplementary material, which is available to authorized users.

$\triangle$ Manabu Takamatsu

manabu.takamatsu@jfcr.or.jp

1 Division of Pathology, The Cancer Institute; Department of Pathology, The Cancer Institute Hospital, Japanese Foundation for Cancer Research, Tokyo 135-8550, Japan

2 Department of Endoscopy, The Cancer Institute Hospital, Japanese Foundation for Cancer Research, Tokyo 135-8550, Japan

3 Department of Colorectal Surgery, The Cancer Institute Hospital, Japanese Foundation for Cancer Research, Tokyo 135-8550, Japan increased over the last few decades [1,2]. Because T1 colorectal cancer can metastasize to lymph nodes with an incidence ranging from $6.3 \%$ to $14.3 \%$ [3-7], strict histologic evaluation of endoscopically resected specimens is necessary for deciding further treatment strategies. Several histologic factors have been proposed for evaluating the risk of lymph node metastasis of $\mathrm{T} 1$ colorectal cancer, with positive lymphatic invasion, positive venous invasion, positive poorly differentiated clusters, highgrade tumor budding, and deep $(\geq 1000 \mu \mathrm{m})$ submucosal invasion as the major risk factors of lymph node metastasis [4, 6-9].

Tumor budding is a well-known prognostic indicator in early and advanced colorectal cancer. Several studies, most of which used hematoxylin and eosin staining for evaluation, have reported the importance of tumor budding [7, 8, 10-13]. Although tumor budding can be evaluated by hematoxylin and eosin staining in some cases, a prominent inflammatory reaction in particular can 
make their observation difficult. Cytokeratin immunohistochemistry can also be used to visualize single cancer cells or buds and may allow for more accurate identification of tumor budding than hematoxylin and eosin staining. Although some studies have investigated the cut-off values between low- and high-grade tumor budding in advanced colorectal cancer using immunohistochemistry $[14,15]$, the optimal cut-off value for stratifying $\mathrm{T} 1$ colorectal cancer has not yet been determined.

In clinical practice, inter-observer reproducibility is another important issue to be considered for efficient prediction of lymph node metastasis. Previous studies compared interobserver variability in budding counts on hematoxylin and eosin- and cytokeratin immunohistochemistry-stained specimens, and the degree of inter-observer agreement as calculated by kappa coefficients was consistently higher for cytokeratin immunohistochemistry than for hematoxylin and eosin staining $[16,17]$. Although cytokeratin immunohistochemistry seems to be an ideal method for evaluating tumor budding, both in-depth histologic instructions and consensus formation between pathologists are needed for highly reproducible assessment. Most recently, Jepsen et al. reported that tumor budding evaluation with digital image analysis was more reproducible than the conventional method in pT1 and pT2 colorectal cancer [18]. In this context, computer-assisted budding assessment is a potential method for resolving these issues.

Here, we conducted a retrospective study to determine the optimal cut-off value between low- and high-grade tumor budding evaluated with cytokeratin immunohistochemistry for stratifying T1 colorectal cancer. In addition, we developed a computer-assisted semiautomatic evaluation method and compared the efficacy of each predictive factor between the manual and semiautomatic methods.

\section{Materials and methods}

\section{Case recruitment and sample preparation}

Five hundred and twenty consecutive T1 colorectal cancer cases from 517 patients resected at The Cancer Institute Hospital between 2005 and 2012, were included in the case review. We adopted both endoscopically $(n=$ $171)$ and surgically $(n=349)$ resected cases to avoid selection biases to determine the actual incidence of lymph node metastasis. Among the patients treated with endoscopic resection, 96 patients $(56.1 \%)$ underwent additional intestinal resection with lymph node dissection, and 74 (43.9\%) patients were followed up by periodic colonoscopy and/or abdominal computed tomography scanning. Serial $2-\mathrm{mm}$ to 5 -mm thick tissue sections of the whole lesion were cut from resected specimens fixed with $20 \%$ buffered formalin and embedded in paraffin, and then $3-\mu \mathrm{m}$ thick sections were prepared for staining. Each section was stained with hematoxylin and eosin. After the initial assessment, 57 cases failed to complete the histologic evaluation due to the sample condition (piece-meal resection, etc.), and eventually 463 cases were included in the study. The cases were split into a derivation dataset $(n=318$, between 2005 and 2010) and a validation dataset $(n=145,2011$ and 2012). All the cases in this study were initially diagnosed by two pathologists (including N.Y.) and then later reviewed by another pathologist (M.T.). Any changes from the initial diagnosis are described in Supplementary Table 1. This study was performed in accordance with the ethics committee of The Cancer Institute Hospital, Japanese Foundation for Cancer Research (Number: 2012-1052).

\section{Histologic assessment and special staining}

One representative section from each case was selected for evaluation of the following factors: tumor depth, lymphatic invasion, venous invasion, poorly differentiated clusters, and tumor budding. Venous invasion was confirmed by hematoxylin and eosin with Victoria-blue staining, which highlights elastic fibers. The depth of submucosal invasion was defined according to the Japanese Society for Cancer of the Colon and Rectum criteria [19]. The poorly differentiated clusters were defined as cancer clusters comprising five or more cells and lacking glandular formation, and regarded as positive if the size was at least $0.24 \mathrm{~mm}^{2}$ using a $\times 40$ objective lens [9]. We evaluated tumor budding using cytokeratin immunohistochemistry (primary antibody: mouse monoclonal anti-cytokeratin AE1/AE3 antibody, clones: AE1 and AE3, in cocktail, Leica Biosystems, Newcastle, UK). We also evaluated the microsatellite instability status. As the majority of sporadic microsatellite instability-high colorectal cancers have lost the expression of MLH1 [20], we evaluated MLH1 expression by immunohistochemistry (primary antibody: mouse monoclonal anti-MLH1 antibody, clone: ES05, Leica Biosystems). The immunohistochemical staining procedures were performed by an auto-stainer (Leica Bond-III, Leica Biosystems). The definition of the tumor budding for manual evaluation was the same as in previous reports [7, 8, 12], which is currently described in the Japanese Society for Cancer of the Colon and Rectum criteria [19]. In brief, tumor budding is a cancer cell or a cluster comprising one to four cells in the invasive frontal region. Pathologists chose the microscopic field using a $\times 20$ objective lens (equal to $0.95 \mathrm{~mm}^{2}$ ) containing the most frequent tumor budding, and counted the number of foci. 


\section{Computer-assisted semiautomatic tumor budding detection}

Automatic detection of tumor budding foci was carried out on micrographs of cytokeratin immunohistochemistrystained sections. Pathologists selected one field containing the most frequent tumor budding focus $\left(0.95 \mathrm{~mm}^{2}\right)$, the same square measure as used for the manual evaluation, and obtained a micrograph of each case. In addition, we considered a different field size $\left(0.785 \mathrm{~mm}^{2}\right)$, according to a previous report [21]. A binary image of each micrograph was generated, and the automatic budding selection was performed according to the size and circularity of the foci. We measured the size $\left(\mu \mathrm{m}^{2}\right)$ of a total of 500 randomly selected cancer clusters (1-5 cells) and non-budding cytokeratin-positive fragments ( 0 cells) to determine thresholds for the automatic selection of budding foci. We also measured the circularity of a total of 100 cytokeratin-positive objects to determine the thresholds. After image processing, manual corrections for eliminating inappropriately selected foci, which were intra-glandular, intra-mucin, glandular, and cytokeratin-negative objects. We defined this evaluation workflow as the semiautomatic method. We used Image J software (National Institute of Health, Bethesda, MD, USA) for image analysis [22]. The workflow from a cytokeratin image to an automatic counting result was executed by macro programming of Image J (Fig. 1a). Macro programming is a series of automated Image $\mathbf{J}$ commands that can be used in the macro editor of Image J. The programming codes are shown in Supplementary Table 2.

\section{Inter-observer agreement study}

Fifty consecutive cases, between December 2007 and August 2008, were selected to evaluate the degree of interobserver reproducibility. Three pathologists (M.T., H.K., and T.M.) evaluated the cases in this study. Budding was graded as low or high according to the optimal cut-off value for cytokeratin immunohistochemistry determined in this study. Prior to evaluation, the pathologists discussed some adoption criteria for budding foci using 10 representative cases that were not part of the 50 consecutive cases. Similarly, the three pathologists and two non-pathologists (M. K.: researcher, and Y.T.: technician) who participated in this study discussed the criteria of the semiautomatic method for 10 representative cases.

\section{Statistical analysis}

The primary endpoint of this study was lymph node metastasis. In cases without surgical treatment, 5-year disease-free survival was regarded as no lymph node metastases. For the derivation dataset, cut-off values of lowor high-grade budding for both the manual and semiautomatic methods were determined by receiver operating characteristic (ROC) curves. The balanced error rate of the ROC curves was considered a candidate value, and the optimal cut-off value was determined based on the odds ratio for lymph node metastasis. The area under the ROC curve (AUC) was considered the predictive ability of the variables, which was ranked as follows: $\leq 0.70$ : low, 0.70-0.90: moderate, and $\geq 0.90$ : high. In the inter-observer agreement study, we used kappa statistics, which can be interpreted as follows: $0.41-0.60$, moderate; $0.61-0.80$, substantial; and $>0.80$, almost perfect, according to a previous study [8].

Univariate and multivariate analyses were conducted for the derivation dataset. In the univariate analyses, the qualitative factors were analyzed with Fischer's exact test, and the quantitative factors were analyzed using unpaired Student's $t$-test with Welch's correction. Multivariate logistic regression was used to calculate the odds ratios and $95 \%$ confidence intervals after simultaneously controlling for potential confounders. Selection of the variables was determined using a stepwise forward selection method, for which $p<0.10$ was considered statistically significant. We analyzed two regression models with the manual and semiautomatic methods, and compared the models using the Akaike information criterion. For the validation dataset, we compared the two methods with calibration plots for predictive and observed frequencies of lymph node metastasis. Correlation of the frequencies was calculated by Pearson's correlation coefficient. We developed a scoring system for the validation dataset depending on the odds ratios of the multivariate analysis, and calculated the scoring points using the approximate ratios. We conducted all analyses using $\mathrm{R}$ version 3.2.4 [23].

\section{Results}

\section{Semiautomatic tumor budding selection}

Representative figures show the workflow of automatic selection, including three inappropriately selected foci eliminated by manual correction (Fig. 1b, c, d and e). The size thresholds to select the budding/sprouting foci comprising one to four cells were $>100 \mu \mathrm{m}^{2}$ and $<480$ $\mu \mathrm{m}^{2}$, with an average size of $253.1 \mu \mathrm{m}^{2}$ (Fig. 1f). The sensitivity and specificity for selecting the foci was approximately $90 \%$ for both the upper and lower thresholds. Because there were two candidate thresholds of circularity, $0.35-1.00$ and $0.45-1.00$, we compared both thresholds using ROC curves. The AUCs were 
Fig. 1 Workflow of semiautomatic tumor budding selection with Image $\mathbf{J}$ software. a Manual and automatic procedures. b Original image of cytokeratin

immunohistochemistry. c Binary image of cytokeratin immunohistochemistry. d Automatically selected budding foci (blue) by size from $100 \mu \mathrm{m}$ to $480 \mu \mathrm{m}$. e Automatically selected tumor budding foci by size and circularity of $0.45-1.00$. Note that some of the foci in (d) are excluded in (e) by low circularity (magnified images shown in the right bottom corner), and some inappropriate foci remain (red objects indicated by red arrows [magnified image shown in the right upper corner]). f Size of cytokeratin-positive objects including 1-5 cancer cells. Small objects not fulfilling the criteria of tumor budding are regarded as 0 cells. Gray zone, from $100 \mu \mathrm{m}^{2}$ to $480 \mu \mathrm{m}^{2}$, was adopted as tumor budding. $\mathbf{g}$ Circularity of true tumor budding and other foci. Gray zone, from 0.45 to 1.00 , was adopted as tumor budding. Scale bars are $100 \mu \mathrm{m}$ a
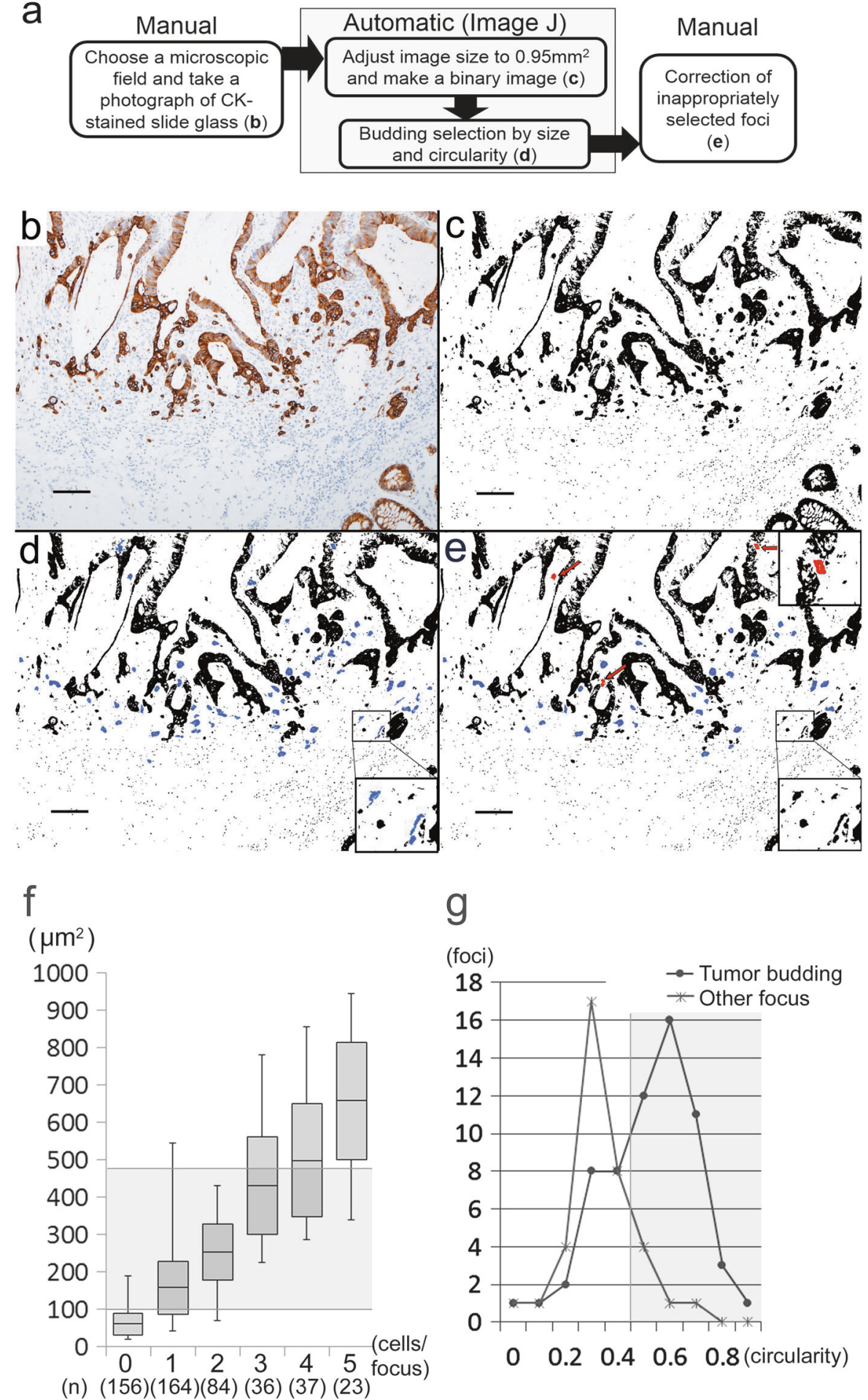

g

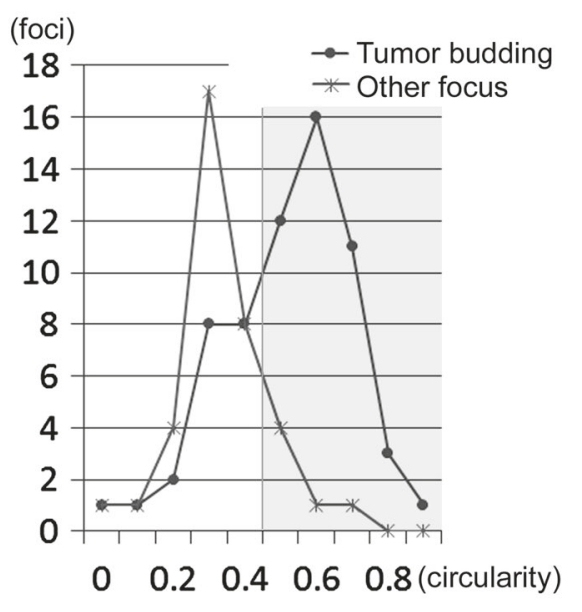

consistently better with manual correction than without it, and almost the same AUCs were observed between 0.35-1.00 and 0.45-1.00 (Supplementary Fig.1a). The latter circularity range showed significantly fewer inappropriately selected foci (Supplementary Fig. 1b), and therefore we applied the threshold of $0.45-1.00$ for the semiautomatic evaluation method (Fig. 1g).

\section{Cut-off value of tumor budding on cytokeratin immunohistochemistry}

Representative figures of tumor budding in the invasive front of T1 colorectal cancer are shown in Fig. 2. The average tumor budding counts on cytokeratin immunohistochemistry-stained sections using the 

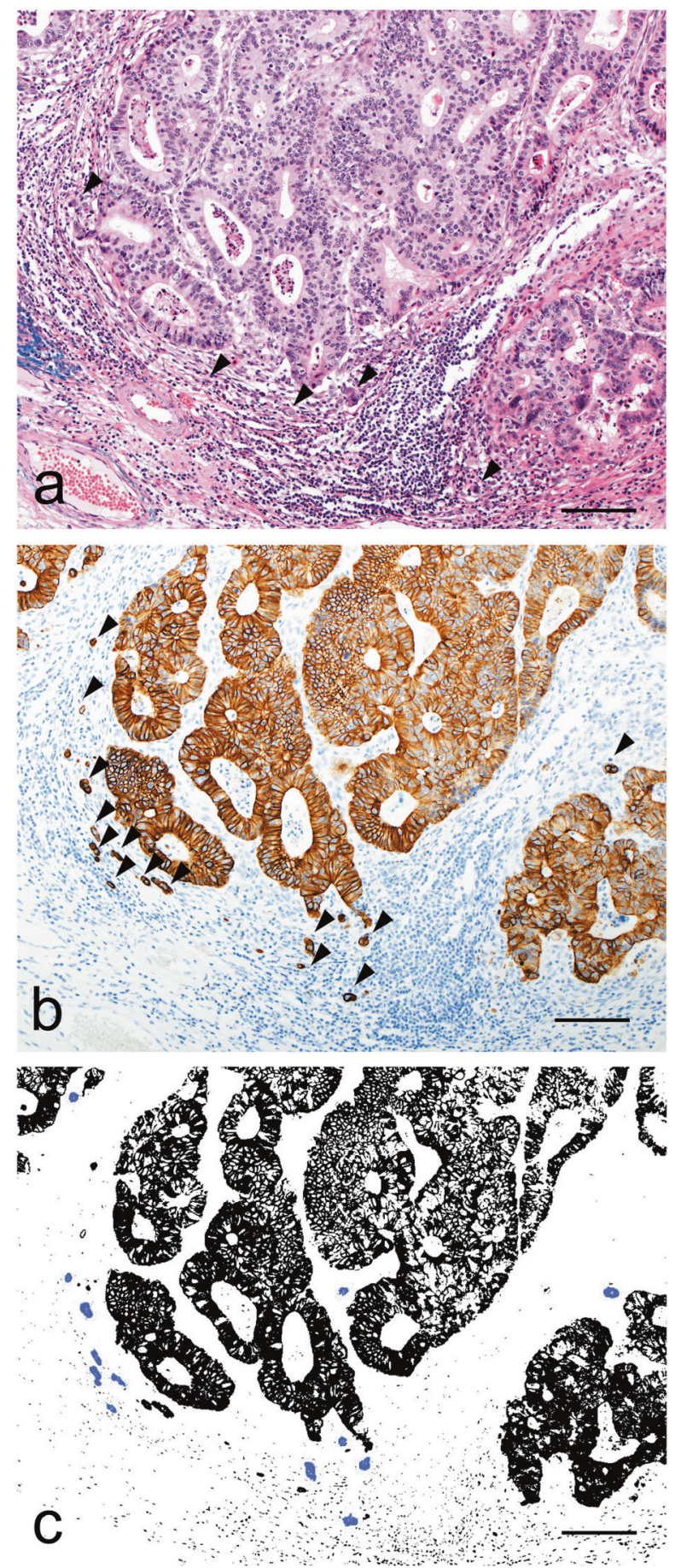

Fig. 2 Representative figures of the invasive front of submucosal invasive colorectal cancer stained with a hematoxylin and eosin and $\mathbf{b}$ cytokeratin immunohistochemistry. The tumor budding count was 5 in hematoxylin and eosin staining, and 14 in cytokeratin immunohistochemistry-stained sections. c Automatic selection of tumor budding, showing 13 identified clusters. Scale bars are $100 \mu \mathrm{m}$

manual and semiautomatic methods were $5.9 \pm 6.4$ and $9.1 \pm 12.6( \pm \mathrm{SD})$, respectively. The average number of manually corrected foci was $1.84 \pm 2.82$
( \pm SD) (Supplementary Fig. 1b). The AUCs of the manual and semiautomatic methods were 0.759 and 0.731 , respectively (Fig. 3). The optimal cut-off values for high-grade budding using the manual and semiautomatic methods were $\geq 10$ and $\geq 12$, respectively. In addition, the same cut-off value, $\geq 12$, was obtained using the semiautomatic method with a $0.785-\mathrm{mm}^{2}$ field size, and the average budding count was $8.4 \pm 11.3( \pm \mathrm{SD}$; Supplementary Fig. 1c). The lymph node metastasis rates for each number of buds are shown in Supplementary Figure 2. These results indicate that both methods showed moderate accuracy for metastatic prediction, and the manual method was slightly better.

\section{Univariate and multivariate analyses of the risk factors for lymph node metastasis}

Table 1 summarizes the results of univariate analyses, and each of the following four factors had a significant influence $(p<0.01)$ on lymph node metastasis: positive lymphatic invasion, positive venous invasion, positive poorly differentiated clusters, and high-grade tumor budding assessed using either the manual or semiautomatic method (Table 1). In multivariate analyses, high-grade tumor budding evaluated using both the manual and semiautomatic methods was an independent risk factor for lymph node metastasis (Table 2). Positive lymphatic invasion and positive venous invasion were also independent risk factors (Table 2). Positive poorly differentiated clusters were not statistically significant. The Akaike information criteria were 158.0 and 163.7 for the manual and semiautomatic methods, respectively. These results indicate that both the manual and semiautomatic methods can be used as predictive indicators for lymph node metastasis, and the manual method was slightly better for the regression model.

\section{Inter-observer agreement was significantly improved with the semiautomatic method}

Among the three pathologists, the average kappa coefficients evaluated with the manual and semiautomatic methods were 0.463 and 0.781 , respectively, and the difference was statistically significant (Fig. 4). Furthermore, among pathologists and non-pathologists, the coefficient was 0.694, indicating substantial interobserver agreement, which was significantly higher than the manual method (Fig. 4). These results indicate that the semiautomatic method can provide reliable evaluative quality, not only by pathologists but also by nonpathologists. 

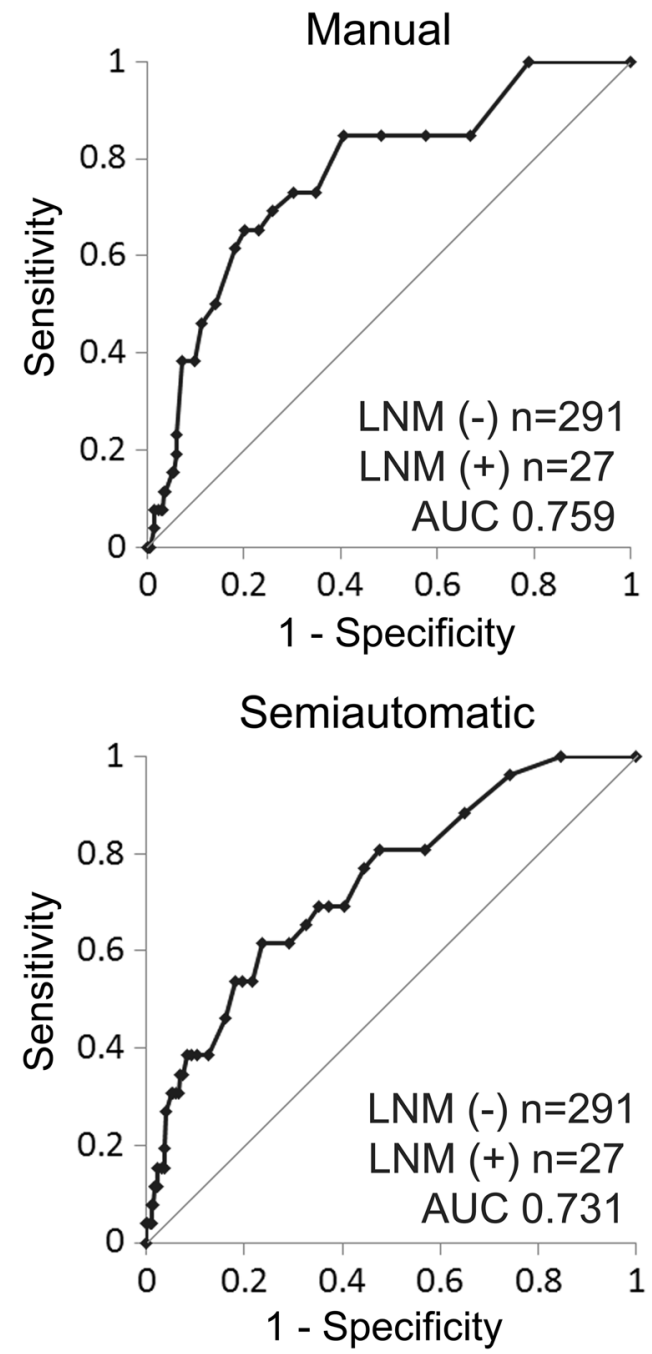

Fig. 3 Receiver operating characteristics (ROC) curves for determining the cut-off values of high-grade tumor budding. Areas under the ROC curves analyzed by manual and semiautomatic methods are 0.759 and 0.731 , respectively

\section{Excellent lymph node metastasis prediction for the validation dataset}

In the validation dataset, the correlation coefficients between predicted and observed lymph node metastases assessed by the models with manual and semiautomatic methods were 0.908 and 0.929 , respectively. Calibration plots for regression models using both methods showed excellent correlation between predicted and observed metastatic frequencies (Fig. 5). We determined the scoring points based on the results of multivariate analysis, as follows: 3 points for lymphatic invasion, 2 points for venous invasion, 3 points for high-grade tumor budding with the manual method, and 2 points for high-grade budding with the semiautomatic method. As a result, similar lymph node metastasis rates were obtained by both methods (Table 3 ).
Table 1 Univariate analysis of features for lymph node metastasis

\begin{tabular}{|c|c|c|c|c|}
\hline Feature & $\begin{array}{l}\text { Negative for } \\
\text { lymph node } \\
\text { metastasis } \\
(\%)\end{array}$ & $\begin{array}{l}\text { Positive for } \\
\text { lymph node } \\
\text { metastasis } \\
(\%)\end{array}$ & $\begin{array}{l}\text { Percentage } \\
\text { of each } \\
\text { criterion }\end{array}$ & $P$-value \\
\hline $\begin{array}{l}\text { Number of } \\
\text { cases (total } n \\
=318 \text { ) }\end{array}$ & 291 & 27 & & \\
\hline \multicolumn{5}{|l|}{ Sex } \\
\hline Male & $166(57)$ & $16(59)$ & $57 \%$ & 1.00 \\
\hline Female & $125(43)$ & $11(41)$ & $43 \%$ & \\
\hline Age & $63.8 \pm 12.0$ & $61.7 \pm 11.3$ & & 0.19 \\
\hline $\begin{array}{l}\text { Tumor size } \\
(\mathrm{mm})\end{array}$ & $20.5 \pm 11.7$ & $23.7 \pm 10.1$ & & 0.08 \\
\hline $\begin{array}{l}\text { Distance to the } \\
\text { vertical margin } \\
(\mathrm{mm}) \\
\text { (Endoscopic } \\
\text { resection only) }\end{array}$ & $\begin{array}{l}1.44 \pm 1.84 \\
(n=99)\end{array}$ & $\begin{array}{l}0.27 \pm 0.39 \\
(n=4)\end{array}$ & & 0.21 \\
\hline \multicolumn{5}{|l|}{ Tumor location } \\
\hline $\mathrm{C}, \mathrm{A}, \mathrm{T}$ & $102(35)$ & $4(15)$ & $33 \%$ & 0.02 \\
\hline $\mathrm{D}, \mathrm{S}, \mathrm{R}$ & $189(65)$ & $23(85)$ & $67 \%$ & \\
\hline \multicolumn{5}{|l|}{ MLH1 expression } \\
\hline Not lost & 277 & 26 & $95 \%$ & 1.00 \\
\hline Lost & 14 & 1 & $5 \%$ & \\
\hline \multicolumn{5}{|c|}{ Depth of submucosal invasion } \\
\hline$<1000 \mu \mathrm{m}$ & $52(18)$ & $1(4)$ & $17 \%$ & 0.06 \\
\hline$\geq 1000 \mu \mathrm{m}$ & $239(82)$ & $26(96)$ & $83 \%$ & \\
\hline \multicolumn{5}{|c|}{ Lymphatic invasion } \\
\hline Negative & $266(91)$ & $16(59)$ & $89 \%$ & $<0.0001$ \\
\hline Positive & $25(9)$ & $11(41)$ & $11 \%$ & \\
\hline \multicolumn{5}{|l|}{ Venous invasion } \\
\hline Negative & $189(65)$ & $9(33)$ & $62 \%$ & $<0.01$ \\
\hline Positive & $102(35)$ & $18(67)$ & $38 \%$ & \\
\hline \multicolumn{5}{|c|}{ Poorly-differentiated clusters } \\
\hline Absent & $275(95)$ & $19(70)$ & $92 \%$ & $<0.001$ \\
\hline Present & $16(5)$ & $8(30)$ & $8 \%$ & \\
\hline \multicolumn{5}{|c|}{ Tumor budding-Manual } \\
\hline $\begin{array}{l}<10 \text { (Low- } \\
\text { grade) }\end{array}$ & $231(79)$ & $10(37)$ & $76 \%$ & $<0.0001$ \\
\hline $\begin{array}{l}\geq 10 \text { (High- } \\
\text { grade) }\end{array}$ & $60(21)$ & $17(63)$ & $24 \%$ & \\
\hline \multicolumn{5}{|c|}{ Tumor budding-Semiautomatic } \\
\hline $\begin{array}{l}<12 \text { (Low- } \\
\text { grade) }\end{array}$ & $222(76)$ & $11(41)$ & $73 \%$ & $<0.001$ \\
\hline $\begin{array}{l}\geq 12 \text { (High- } \\
\text { grade) }\end{array}$ & $69(24)$ & $16(59)$ & $27 \%$ & \\
\hline
\end{tabular}

$C$ cecum, $A$ ascending colon, $T$ transverse colon, $D$ descending colon, $S$ sigmoid colon, $R$ rectum

These results indicate that both manual and semiautomatic methods can effectively predict lymph node metastasis, and the model using the semiautomatic method was slightly better. 
Table 2 Multivariate analysis of the four parameters using a logistic regression model

\begin{tabular}{|c|c|c|}
\hline Parameter & $\begin{array}{l}\text { Odds ratio }(95 \% \\
\text { confidence interval) }\end{array}$ & $P$-value \\
\hline \multicolumn{3}{|l|}{ Manual method } \\
\hline Lymphatic invasion positive & $4.4(1.7-11.0)$ & $<0.01$ \\
\hline Venous invasion positive & $2.8(1.2-6.9)$ & 0.02 \\
\hline $\begin{array}{l}\text { Tumor budding }- \text { Manual } \geq 10 \\
\text { (High-grade) }\end{array}$ & $4.1(1.7-10.0)$ & $<0.01$ \\
\hline $\begin{array}{l}\text { Poorly differentiated } \\
\text { components present }\end{array}$ & N.A. & N.A. \\
\hline \multicolumn{3}{|l|}{ Semiautomatic method } \\
\hline Lymphatic invasion positive & $4.6(1.8-11.7)$ & $<0.01$ \\
\hline Venous invasion positive & $2.7(1.1-6.6)$ & 0.03 \\
\hline $\begin{array}{l}\text { Tumor budding - } \\
\text { Semiautomatic } \geq 12 \text { (High- } \\
\text { grade) }\end{array}$ & $2.6(1.1-6.3)$ & 0.03 \\
\hline $\begin{array}{l}\text { Poorly differentiated } \\
\text { components present }\end{array}$ & N.A. & N.A. \\
\hline
\end{tabular}

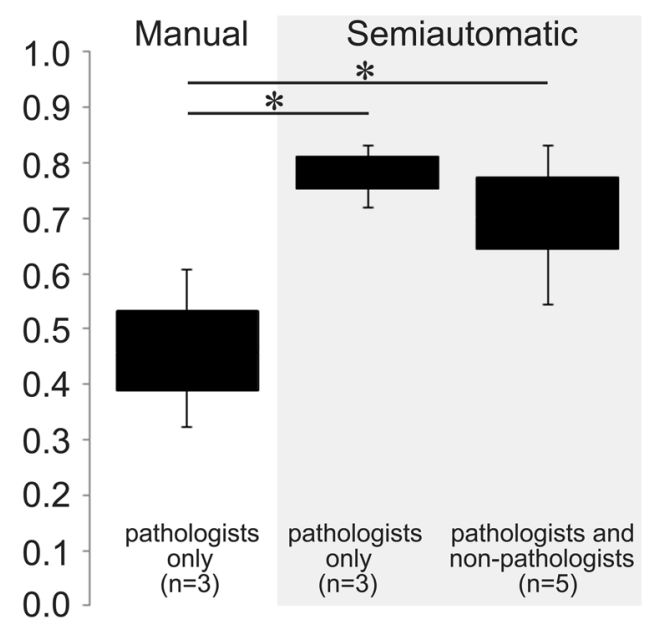

Fig. 4 Average kappa coefficient in the inter-observer agreement study. Among three pathologists, the average kappa coefficient was significantly higher with the semiautomatic method than the manual method. Similarly, among three pathologists and two non-pathologists, the coefficient was significantly higher with the semiautomatic method than the manual method. $* p<0.05$ by Student's $t$-test

\section{Discussion}

In the present study, we determined the optimal cut-off value for high-grade tumor budding for $\mathrm{T} 1$ colorectal cancer on cytokeratin immunohistochemistry-stained sections, and established a computer-assisted semiautomatic evaluation method with high reproducibility. Conventional histologic evaluation of tumor budding by hematoxylin and eosin staining is well investigated. The most widely accepted definition of tumor budding is a cell cluster comprising fewer than five cells $[7,8,12]$. The Japanese Society for
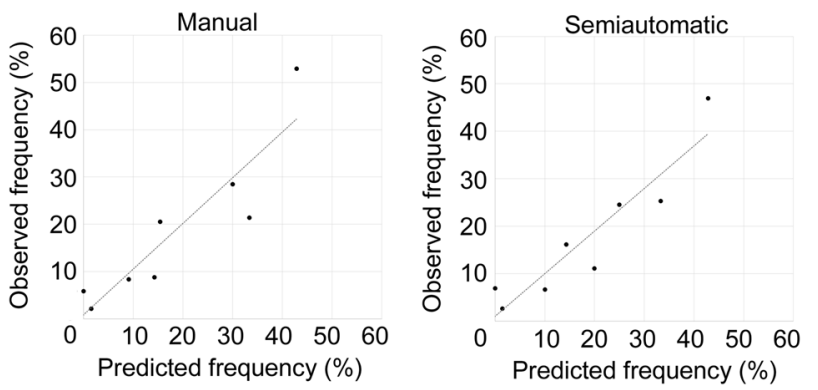

Fig. 5 Calibration plots for the validation dataset. Each dot represents lymph node metastasis frequency of cases with a different combination of risk factors. Both models with manual and semiautomatic methods showed excellent prediction for lymph node metastasis

Table 3 Predictive simulation for the validation dataset

\begin{tabular}{|c|c|c|c|}
\hline $\begin{array}{l}\text { Total } \\
\text { points }\end{array}$ & $\begin{array}{l}\text { Number of } \\
\text { cases }\end{array}$ & $\begin{array}{l}\text { Positive for lymph } \\
\text { node metastasis }\end{array}$ & $\begin{array}{l}\text { Rate of lymph } \\
\text { node metastasis }\end{array}$ \\
\hline \multicolumn{4}{|c|}{ Manual method } \\
\hline 0 & 65 & 1 & $1.5 \%$ \\
\hline 2 & 18 & 0 & $0.0 \%$ \\
\hline 3 & 29 & 3 & $10.3 \%$ \\
\hline 5 & 16 & 3 & $18.8 \%$ \\
\hline 6 & 10 & 3 & $30.0 \%$ \\
\hline 8 & 7 & 3 & $42.9 \%$ \\
\hline \multicolumn{4}{|c|}{ Semiautomatic method } \\
\hline 0 & 67 & 1 & $1.5 \%$ \\
\hline 2 & 37 & 2 & $5.4 \%$ \\
\hline 3 & 5 & 1 & $20.0 \%$ \\
\hline 4 & 14 & 2 & $14.3 \%$ \\
\hline 5 & 15 & 4 & $26.7 \%$ \\
\hline 7 & 7 & 3 & $42.9 \%$ \\
\hline
\end{tabular}

Points for manual method: 3 points for positive lymphatic invasion and high-grade tumor budding, 2 points for positive venous invasion.

Points for semiautomatic method: 3 points for positive lymphatic invasion, 2 points for positive venous invasion and high-grade tumor budding

Cancer of the Colon and Rectum guideline for T1 colorectal cancer defines high-grade budding (Grades 2 and 3 ) as 5 or more foci in the field of a $\times 20$ objective lens [19]. According to this guideline, the grade of tumor budding should be described in routine pathology reports for $\mathrm{T} 1$ colorectal cancer, but the recommended evaluation method (e.g., hematoxylin and eosin staining only) is not described. On the other hand, the International Tumor Budding Consensus Conference group strongly recommended that tumor budding should be evaluated on hematoxylin and eosin, since the majority of the studies were based on hematoxylin and eosin staining [21]. Some studies have explored the importance of tumor budding evaluated by cytokeratin immunohistochemistry. Ohtsuki et al. reported that a significantly higher budding count was obtained by cytokeratin 
immunohistochemistry than by hematoxylin and eosin staining in advanced colorectal cancer, and encouraged the use of immunohistochemistry for more accurate prediction of disease-free survival [24]. In daily clinical practice, cytokeratin immunohistochemistry is performed for $\mathrm{T} 1$ colorectal cancer with obscure budding foci, especially in cases with prominent inflammation to avoid misidentifying some plump nuclei of fibroblasts or endothelial cells as budding [25]. Cytokeratin immunohistochemistry is helpful for distinguishing true tumor budding from other cells, but an inaccurately high budding count may lead to overestimation of the budding grade and unnecessary additional surgery. In this study, we determined the optimal cut-off value for cytokeratin immunohistochemistry by manual evaluation as $\geq 10$, which was twice as high as the value for evaluation with hematoxylin and eosin staining. Our results clearly indicate that different cut-off values should be applied to cases evaluated with cytokeratin immunohistochemistry in $\mathrm{T} 1$ colorectal cancer.

Inter-observer agreement for tumor budding related to cytokeratin immunohistochemistry was described in previous studies. Kai et al. described the usefulness of immunohistochemistry for unskilled pathologists to improve the agreement compared with hematoxylin and eosin staining [17]. A recent report described that immunohistochemical budding evaluation is not an independent risk factor for lymph node metastasis of $\mathrm{T} 1$ colorectal cancer, and the cutoff value of high-grade tumor budding on cytokeratin immunohistochemistry-stained sections was 8 [26], which substantially differs from our result. This discrepancy may be due to the interpretation gap among pathologists for cytokeratin-positive foci. These facts indicate that sufficient inter-observer consensus formation is necessary before clinical application of the manual methods. The novel semiautomatic method proposed in this study can provide a highly reproducible budding evaluation with only 10 cases for substantial consensus formation, not only by pathologists but also non-pathologists. This method provides better reproducibility than conventional methods, requiring only cytokeratin immunohistochemistry and a computer with Image $\mathbf{J}$ software, which is universally available.

Our semiautomatic method can be accomplished using a single micrograph and a simple computer operation with manual corrections, which is a better tool for evaluating tumor budding than the conventional method. The method takes only a few minutes to perform, and can thus be readily used in daily diagnostic practice with the freely available Image J program and preinstalled macros. A fully automatic method, however, is preferred to completely avoid investigator error. In our semiautomatic method, the computer software selected some inappropriate objects, which resulted in false-positive clusters. In addition, a single cancer cell smaller than $100 \mu^{2}$ was neglected in the semiautomatic selection, which resulted in the elimination of true tumor budding and was considered a false-negative focus. Similarly, tumor clusters over $480 \mu^{2}$ in size were also neglected. Because of these objects, the predictive value might not be superior to manual evaluation. A previous study reported similar problems with false-negative and false-positive objects, which required manual adjustment for efficient prediction of lymph node metastasis [18]. We used freely available Image $\mathbf{J}$ software and adopted only two parameters for selecting tumor budding foci, size, and circularity; hence, the method is simple and has universal applicability. Further development of the computer software is needed for full-automation.

In summary, a proper cut-off value should be applied for evaluating tumor budding of $\mathrm{T} 1$ colorectal cancer with cytokeratin immunohistochemistry. The semiautomatic method proposed in this study may be a useful alternative for highly reproducible tumor budding evaluation in clinical practice.

Acknowledgements The authors thank Ms. Tomoyo Kakita, Mr. Motoyoshi Iwakoshi, and Ms. Miki Hatta for excellent technical assistance.

\section{Compliance with ethical standards}

Conflict of interest The authors declare that they have no conflict of interest.

\section{References}

1. Marin-Gabriel JC, Fernandez-Esparrach G, Diaz-Tasende J, et al. Colorectal endoscopic submucosal dissection from a Western perspective: Today's promises and future challenges. World J Gastrointest Endosc. 2016;8:40-55.

2. Urabe Y, Tanaka S, Saito Y, et al. Impact of revisions of the JSCCR guidelines on the treatment of T1 colorectal carcinomas in Japan. Z Gastroenterol. 2015;53:291-301.

3. Yamamoto S, Watanabe M, Hasegawa H, et al. The risk of lymph node metastasis in $\mathrm{T} 1$ colorectal carcinoma. Hepatogastroenterology. 2004;51:998-1000.

4. Kitajima K, Fujimori T, Fujii S, et al. Correlations between lymph node metastasis and depth of submucosal invasion in submucosal invasive colorectal carcinoma: a Japanese collaborative study. J Gastroenterol. 2004;39:534-43.

5. Shimomura $\mathrm{T}$, Ishiguro $\mathrm{S}$, Konishi $\mathrm{H}$, et al. New indication for endoscopic treatment of colorectal carcinoma with submucosal invasion. J Gastroenterol Hepatol. 2004;19:48-55.

6. Tateishi Y, Nakanishi Y, Taniguchi H, et al. Pathological prognostic factors predicting lymph node metastasis in submucosal invasive (T1) colorectal carcinoma. Mod Pathol. 2010;23: 1068-72.

7. Kawachi H, Eishi Y, Ueno $\mathrm{H}$, et al. A three-tier classification system based on the depth of submucosal invasion and budding/ sprouting can improve the treatment strategy for $\mathrm{T} 1$ colorectal cancer: a retrospective multicenter study. Mod Pathol. 2015;28: $872-9$.

8. Ueno H, Murphy J, Jass JR, et al. Tumour 'budding' as an index to estimate the potential of aggressiveness in rectal cancer. Histopathology. 2002;40:127-32. 
9. Ueno H, Hashiguchi Y, Kajiwara Y, et al. Proposed objective criteria for "grade 3" in early invasive colorectal cancer. Am J Clin Pathol. 2010;134:312-22.

10. Ha SS, Choi HJ, Park KJ, et al. Intensity of tumor budding as an index for the malignant potential in invasive rectal carcinoma. Cancer Res Treat. 2005;37:177-82.

11. Losi L, Ponti G, Gregorio CD, et al. Prognostic significance of histological features and biological parameters in stage I (pT1 and pT2) colorectal adenocarcinoma. Pathol Res Pract. 2006;202: 663-70.

12. Yamauchi H, Togashi K, Kawamura YJ, et al. Pathological predictors for lymph node metastasis in T1 colorectal cancer. Surg Today. 2008;38:905-10.

13. Karamitopoulou E, Zlobec I, Kolzer V, et al. Proposal for a 10high-power-fields scoring method for the assessment of tumor budding in colorectal cancer. Mod Pathol. 2013;26:295-301.

14. Prall F, Nizze H, Barten M. Tumour budding as prognostic factor in stage I/II colorectal carcinoma. Histopathology. 2005;47: $17-24$.

15. Zlobec I, Molinari F, Martin V, et al. Tumor budding predicts response to anti-EGFR therapies in metastatic colorectal cancer patients. World J Gastroenterol. 2010;16:4823-31.

16. Koelzer VH, Zlobec I, Lugli A. Tumor budding in colorectal cancer-ready for diagnostic practice? Hum Pathol. 2016;47:4-19.

17. Kai K, Aishima S, Aoki S, et al. Cytokeratin immunohistochemistry improves interobserver variability between unskilled pathologists in the evaluation of tumor budding in T1 colorectal cancer. Pathol Int. 2016;66:75-82.
18. Jepsen RK, Klarskov LL, Lippert MF, et al. Digital image analysis of pan-cytokeratin stained tumor slides for evaluation of tumor budding in pT1/pT2 colorectal cancer: Results of a feasibility study. Pathol Res Pract 2018;214:1273-81.

19. Watanabe T, Itabashi M, Shimada Y, et al. Japanese Society for Cancer of the Colon and Rectum (JSCCR) Guidelines 2014 for treatment of colorectal cancer. Int J Clin Oncol. 2015;20:207-39.

20. Boland CR, Goel A. Microsatellite instability in colorectal cancer. Gastroenterology. 2010;138:2073-87. e3

21. Lugli A, Kirsch R, Ajioka Y, et al. Recommendations for reporting tumor budding in colorectal cancer based on the International Tumor Budding Consensus Conference (ITBCC) 2016. Mod Pathol. 2017;30:1299-311.

22. Schneider CA, Rasband WS, Eliceiri KW. NIH Image to ImageJ: 25 years of image analysis. Nat Methods. 2012;9:671-5.

23. R: A language and environment for statistical computing. $\mathrm{R}$ Foundation for Statistical Computing, Vienna, Austria.

24. Ohtsuki K, Koyama F, Tamura T, et al. Prognostic value of immunohistochemical analysis of tumor budding in colorectal carcinoma. Anticancer Res. 2008;28:1831-6.

25. Mitrovic B, Schaeffer DF, Riddell RH, et al. Tumor budding in colorectal carcinoma: time to take notice. Mod Pathol. 2012;25: 1315-25.

26. Okamura T, Shimada Y, Nogami H, et al. Tumor Budding Detection by Immunohistochemical Staining is Not Superior to Hematoxylin and Eosin Staining for Predicting Lymph Node Metastasis in pT1 Colorectal Cancer. Dis Colon Rectum. 2016;59:396-402. 